\title{
Concise and efficient synthesis of [6]-paradol
}

Guangyan Zhang"2\#, TianTian Xia"\#, Brooke E. McKamey ${ }^{3}$, Xian $\mathrm{Wu}^{3}$, Yue Sun ${ }^{4}$, Weifeng Zhou ${ }^{5}$, Xiang Shi ${ }^{1 *}$

${ }^{1}$ State Key Laboratory of Bioelectronics, School of Biological Science and Medical Engineering, Southeast University, Nanjing, Jiangsu 210096, P. R. China.

2 State Key Laboratory of Bioactive Substances and Functions of Natural Medicines, Beijing Key Laboratory of Active Substances Discovery and Druggability Evaluation, Institute of Materia Medica, Chinese Academy of Medical Sciences and Peking Union Medical College, Beijing 100050, China.

${ }^{3}$ Department of Kinesiology, Nutrition and Health, Miami University, Oxford, $\mathrm{OH}$ 45056, USA

${ }^{4}$ Anhui Engineering Laboratory for Agro-products Processing, School of Tea \& Food Science, International Joint Laboratory on Tea Chemistry and Health Effects, Anhui Agricultural University, Hefei, 230036, China

${ }^{5}$ Xuchang Yuanzhi Biological Technology Co., Ltd. Xuchang, Henan 461000, P. R. China. 


\section{Supporting Information.}

1) ${ }^{1} \mathrm{H}$ and ${ }^{13} \mathrm{C}$ NMR spectra of the Compounds $16,17,19,20,1$ and $1 \mathrm{a}$. ${ }^{1} \mathrm{H}$ NMR spectra of Compound 16.

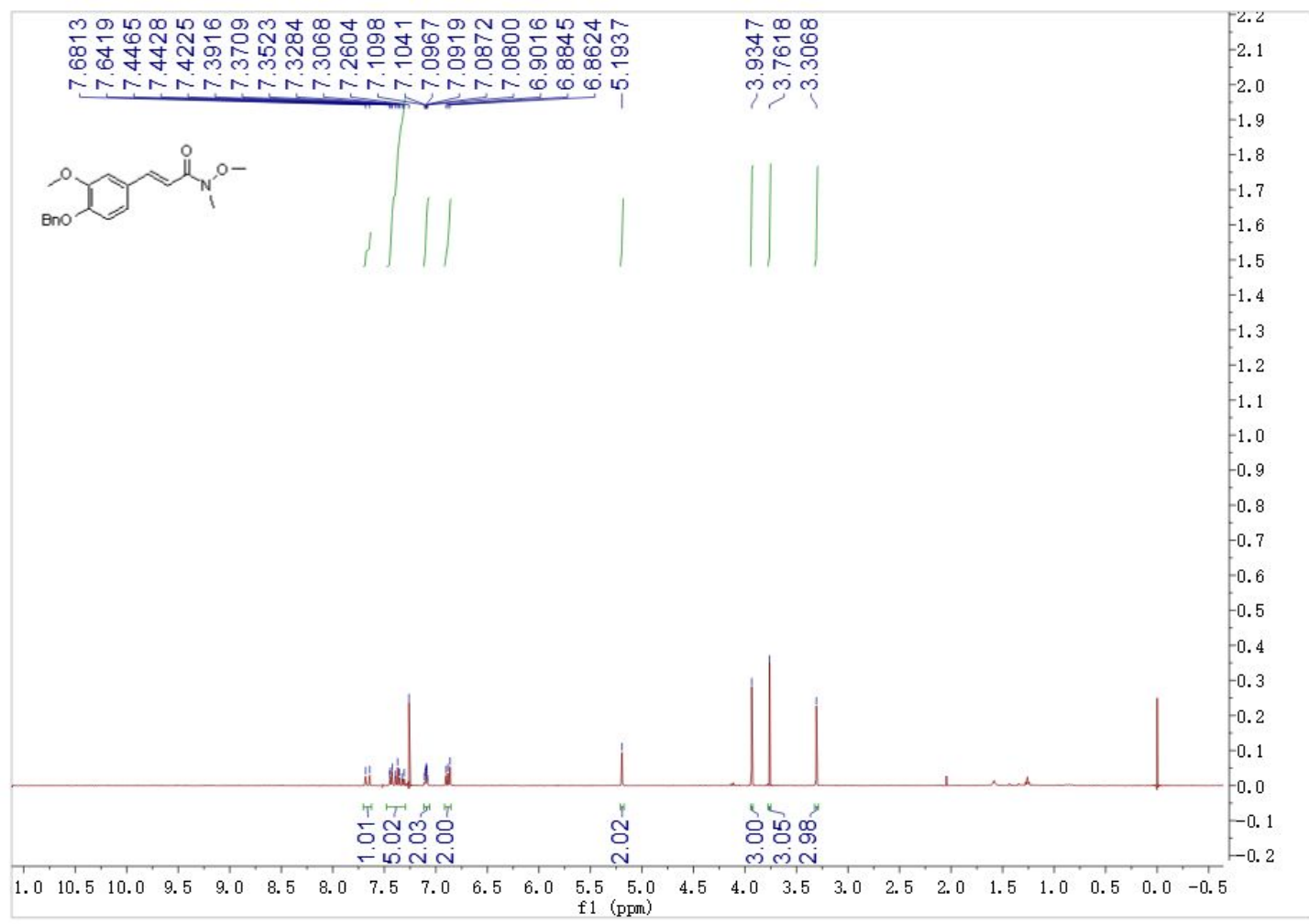

${ }^{13} \mathrm{C}$ NMR spectra of Compound 16.

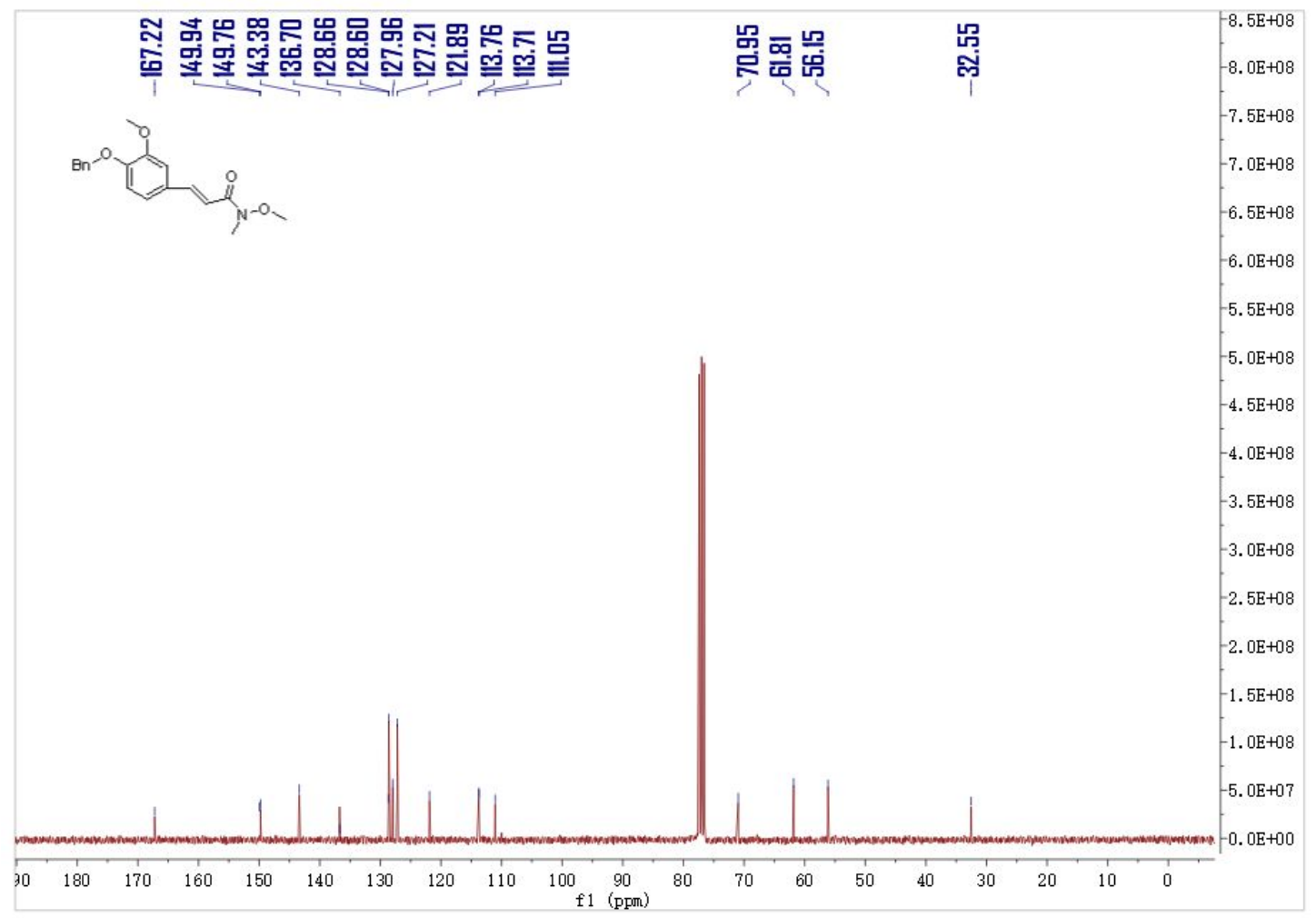


${ }^{1} \mathrm{H}$ NMR spectra of Compound 17.

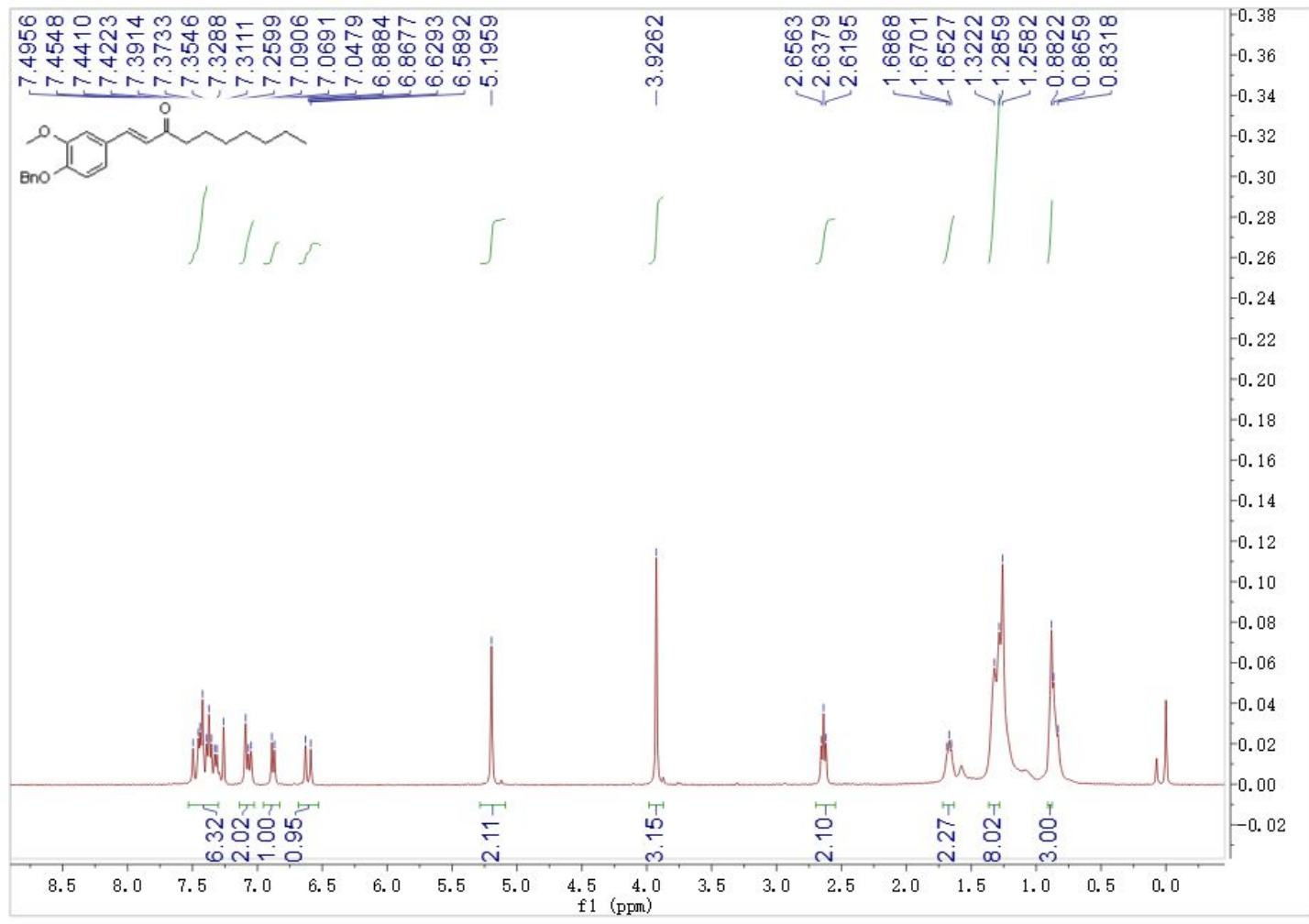

${ }^{13} \mathrm{C}$ NMR spectra of Compound 17.

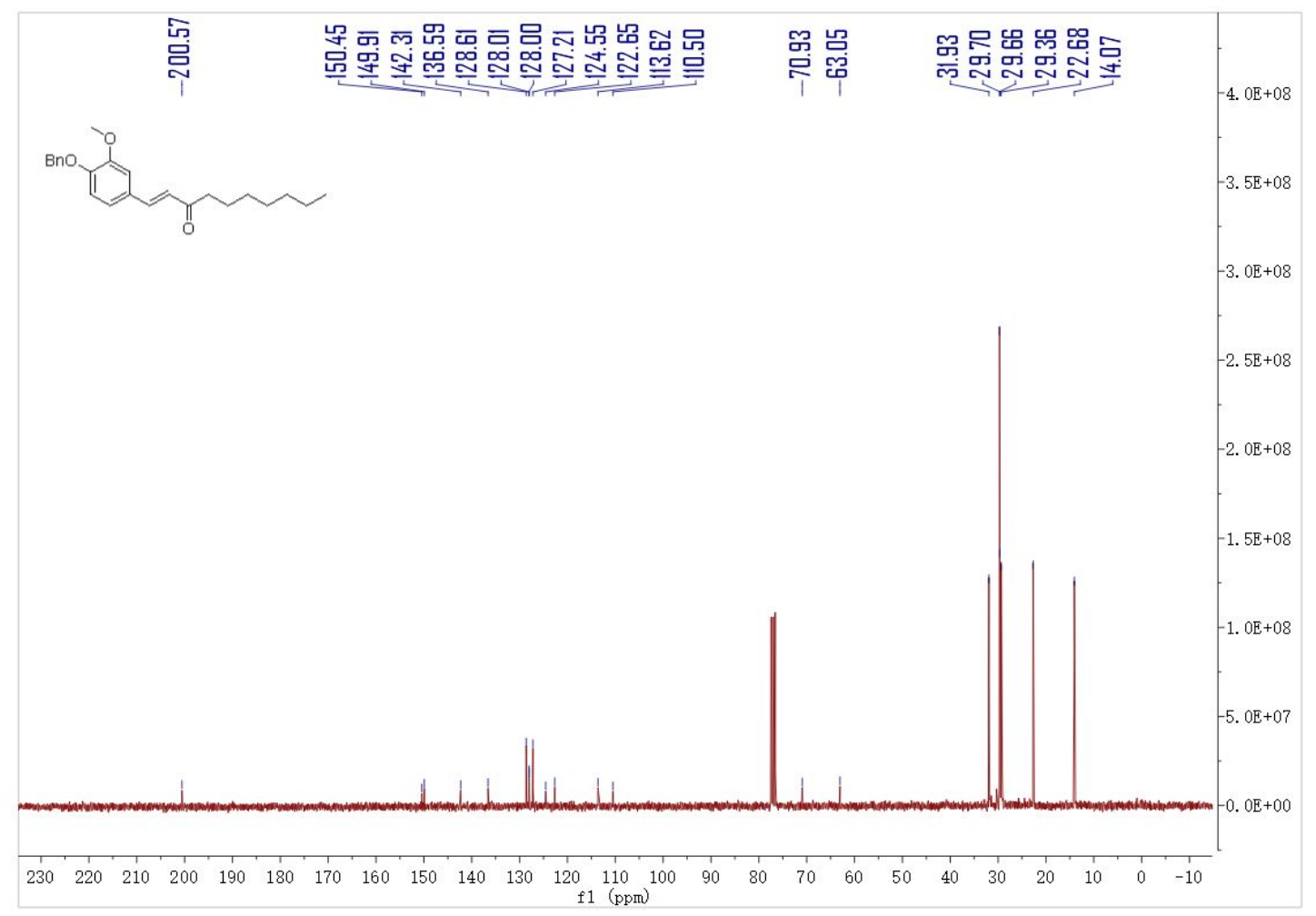


${ }^{1} \mathrm{H}$ NMR spectra of Compound 19.

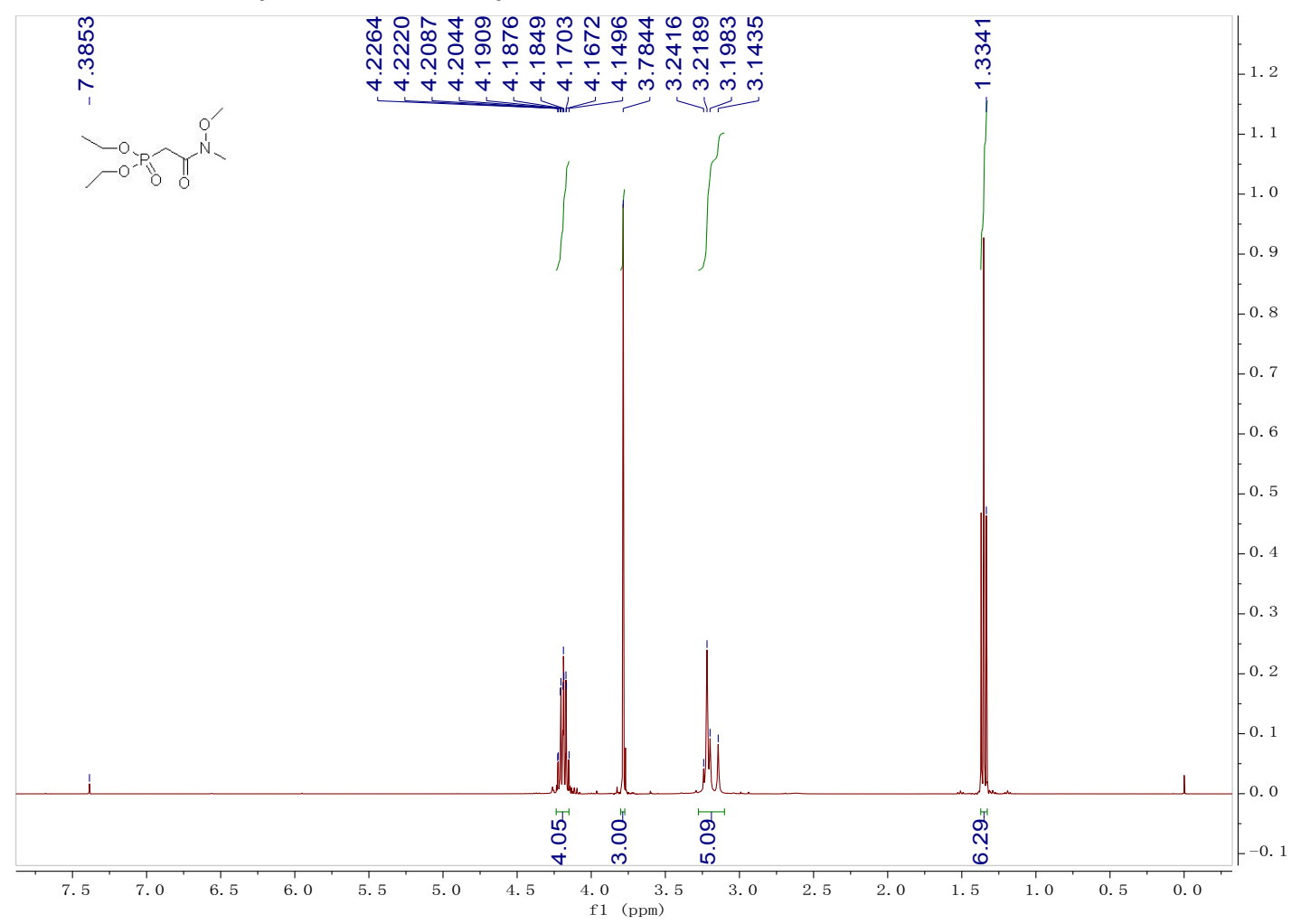

${ }^{13} \mathrm{C}$ NMR spectra of Compound 19.

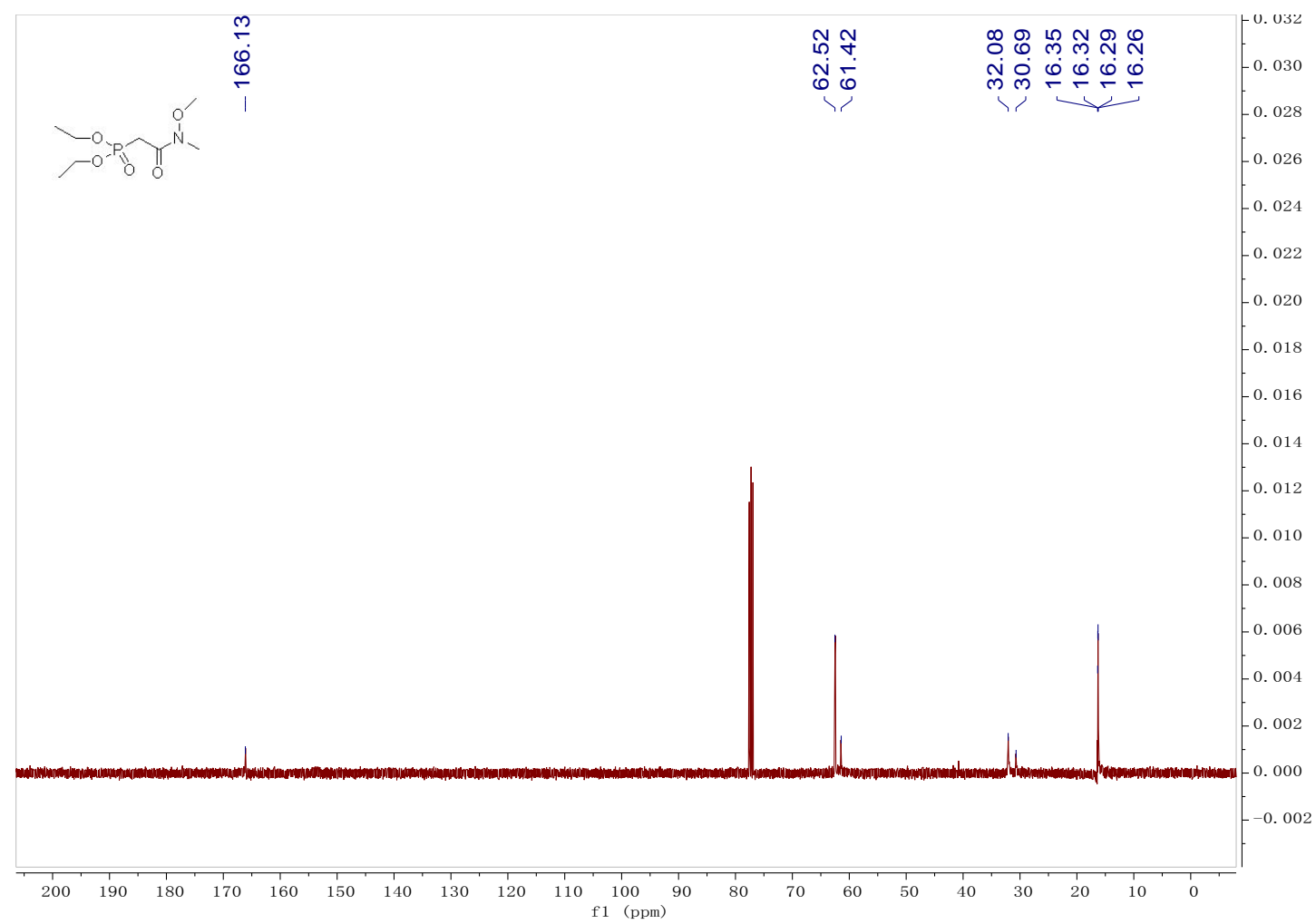


${ }^{1} \mathrm{H}$ NMR spectra of Compound 20.

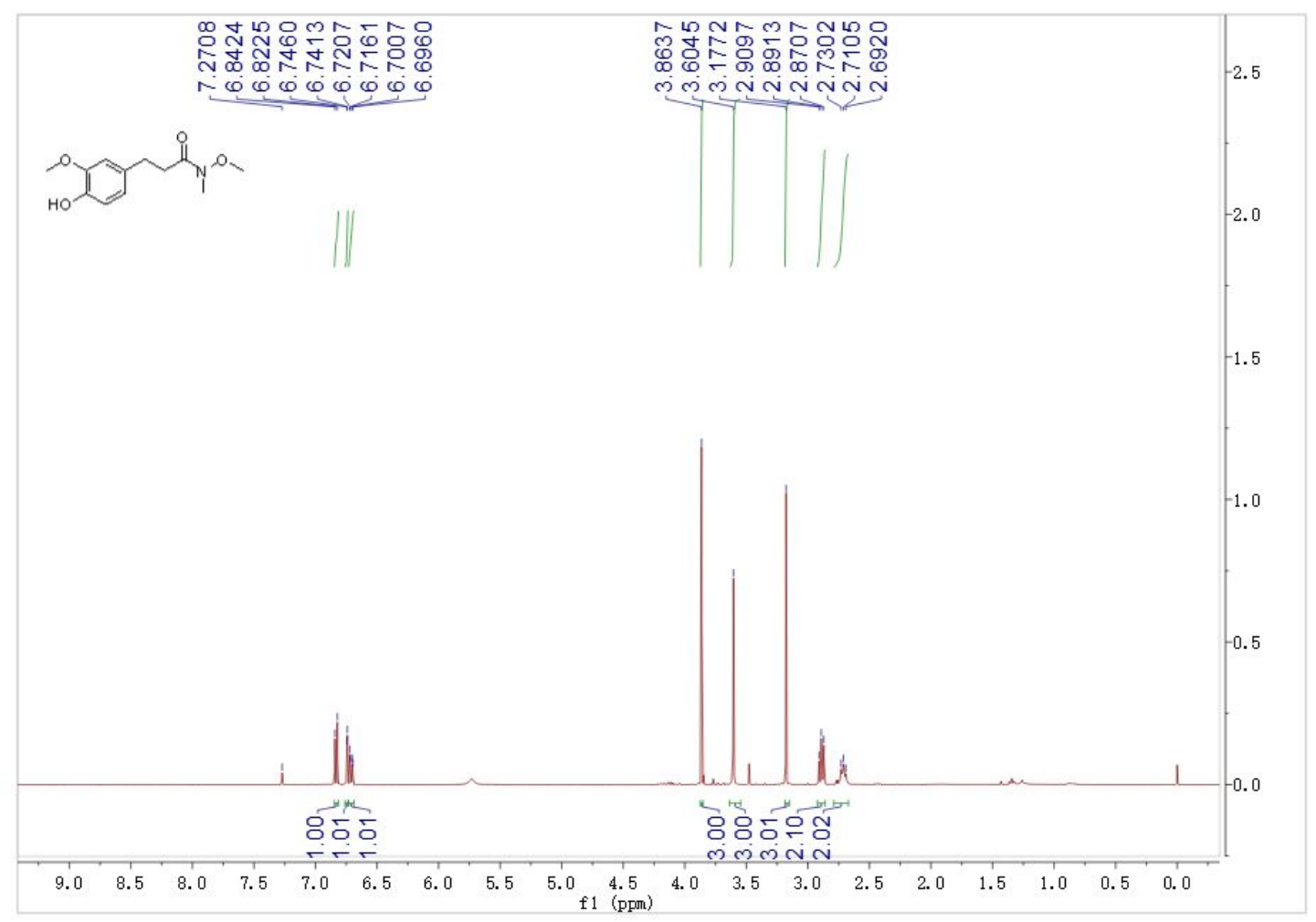

${ }^{13} \mathrm{C}$ NMR spectra of Compound 20.

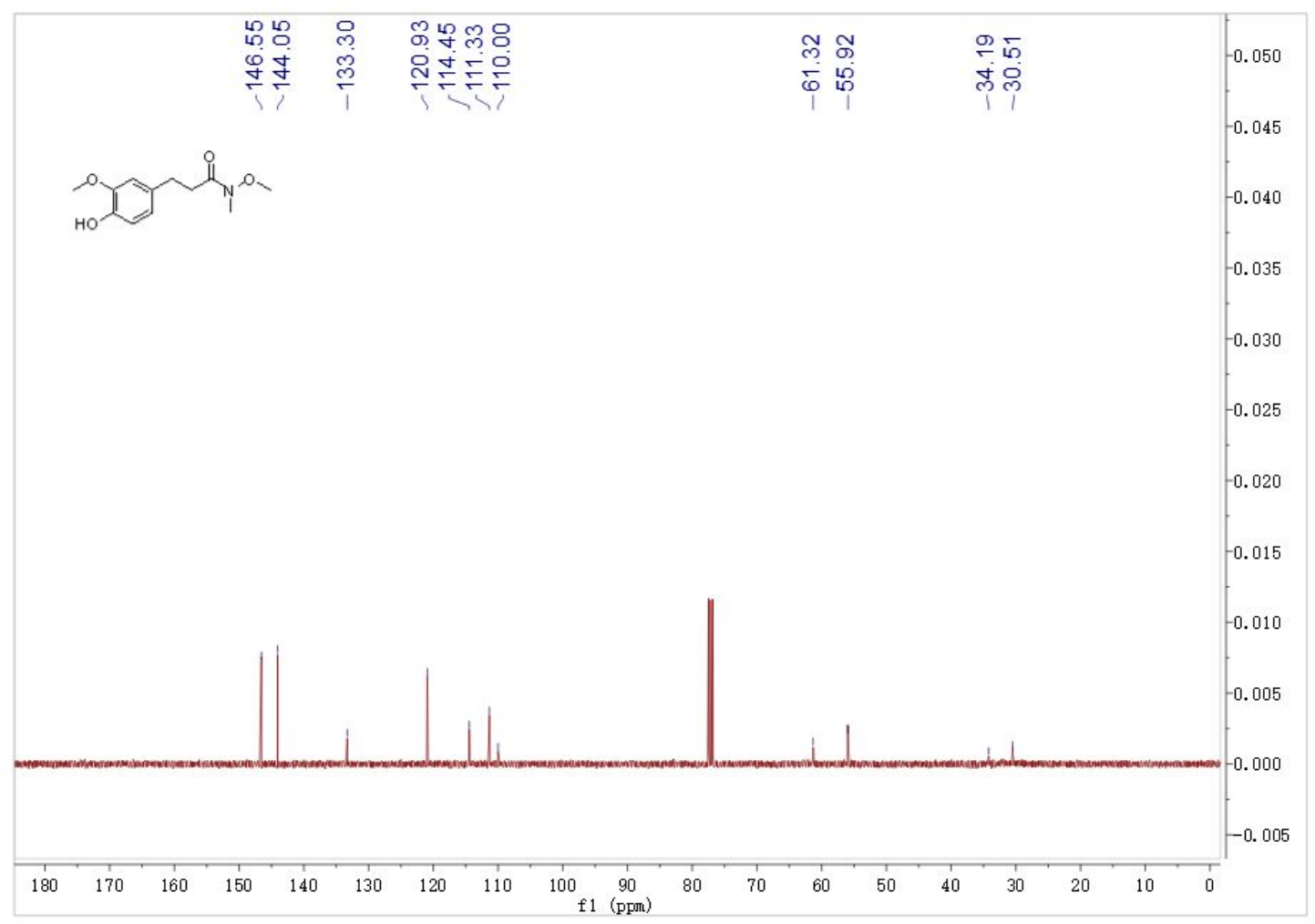


${ }^{1} \mathrm{H}$ NMR spectra of Compound 1.

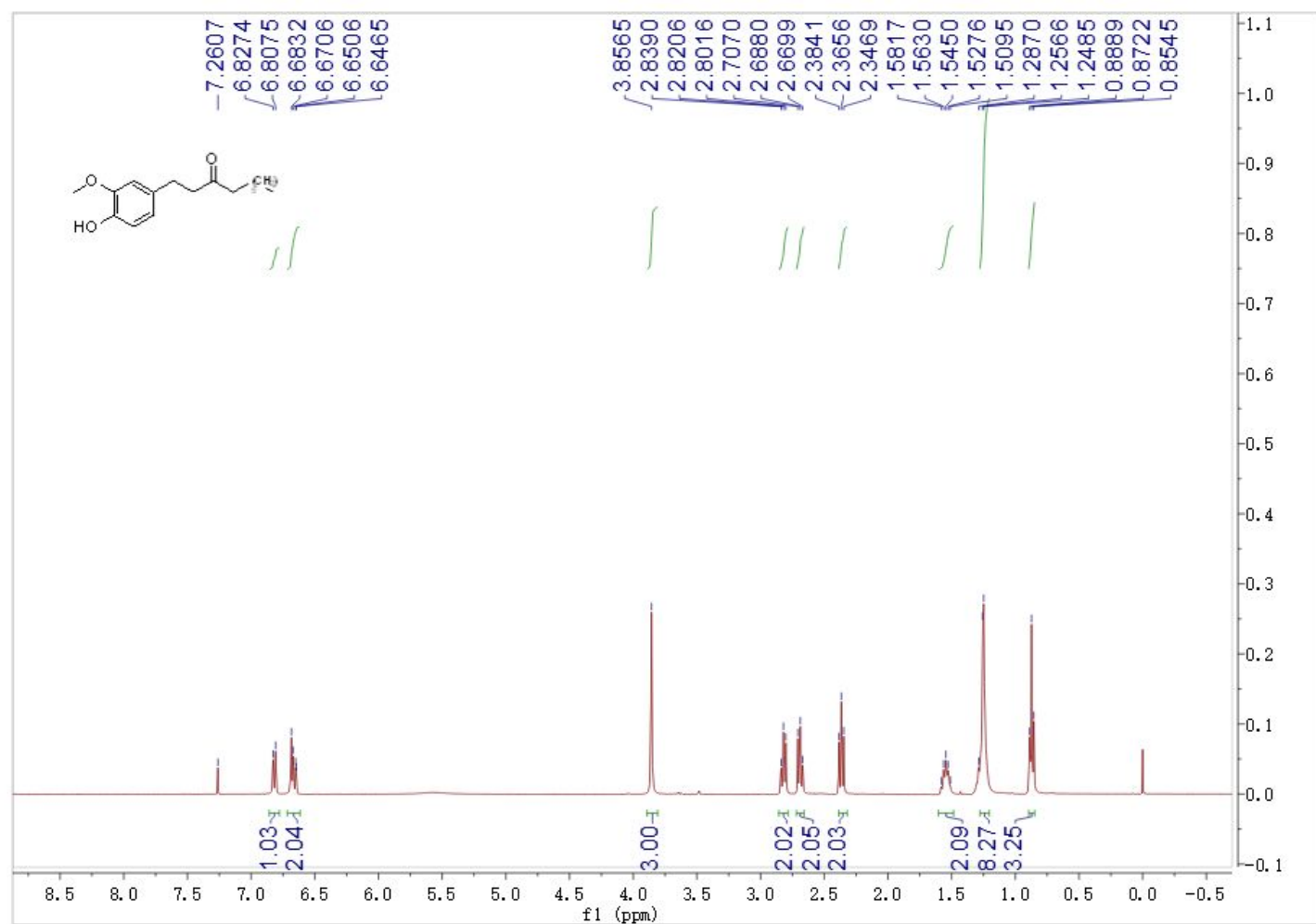

${ }^{13} \mathrm{C}$ NMR spectra of Compound 1.

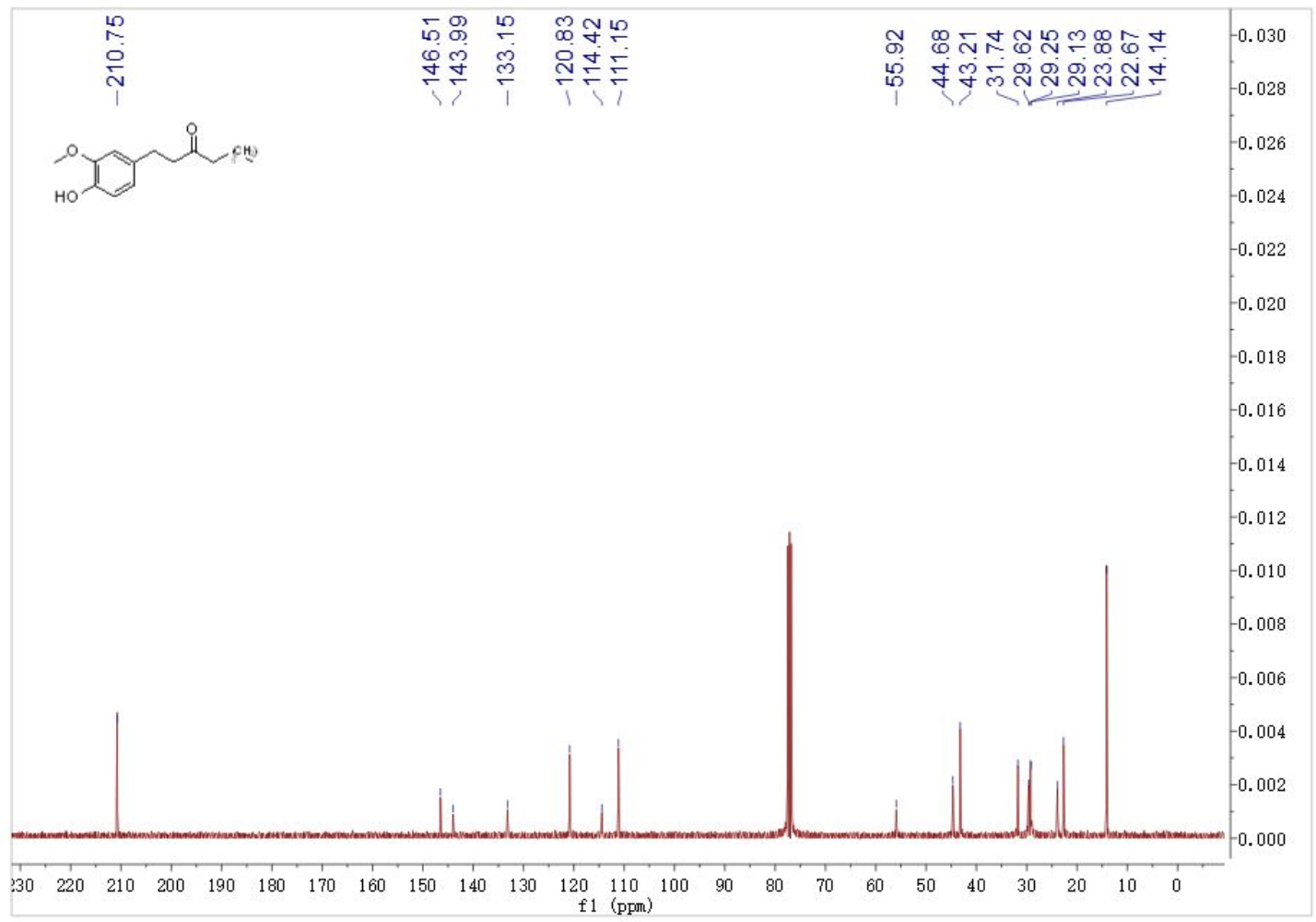


${ }^{1} \mathrm{H}$ NMR spectra of Compound $\mathbf{1 a}$.

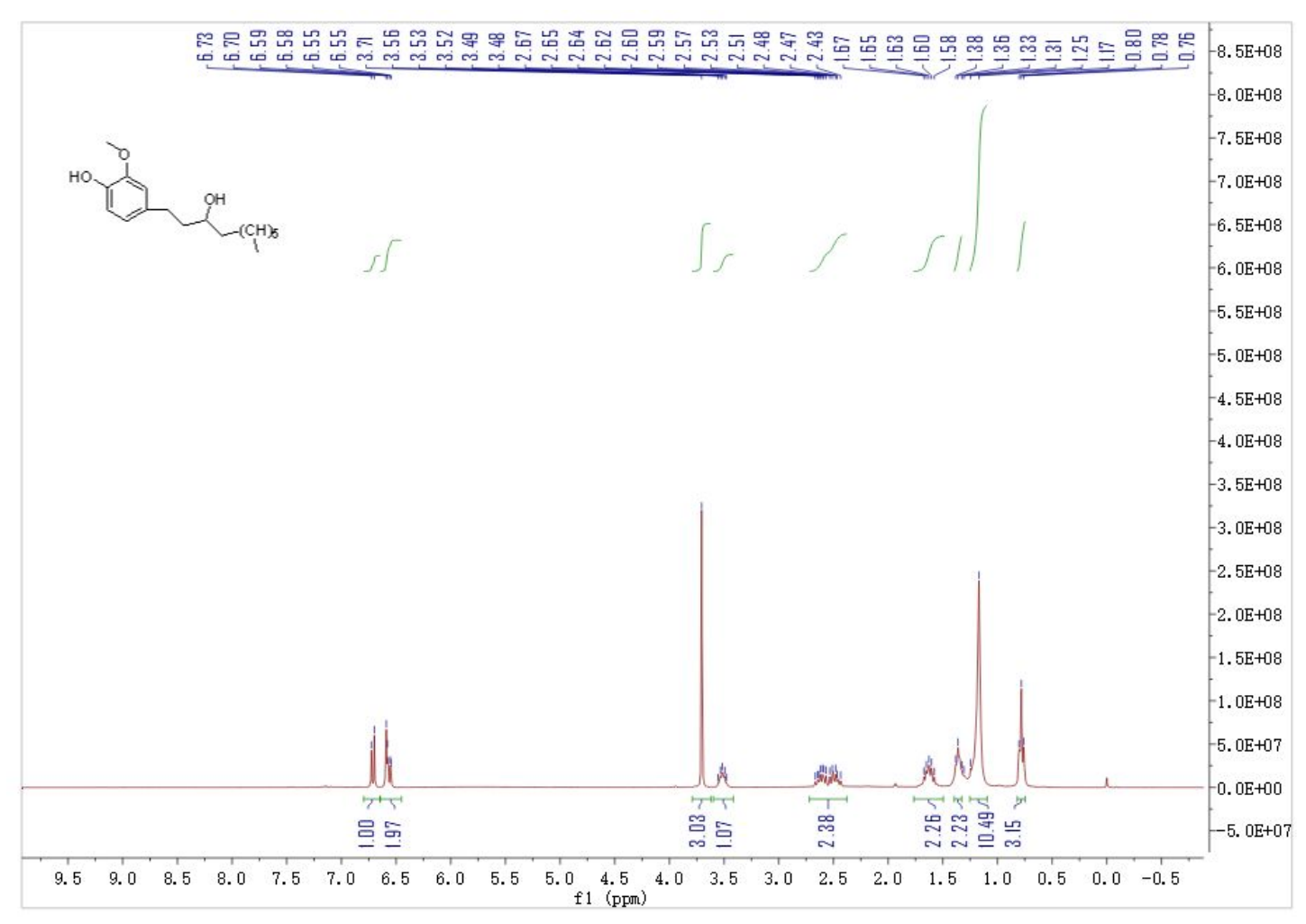

${ }^{13} \mathrm{C}$ NMR spectra of Compound $\mathbf{1 a}$.

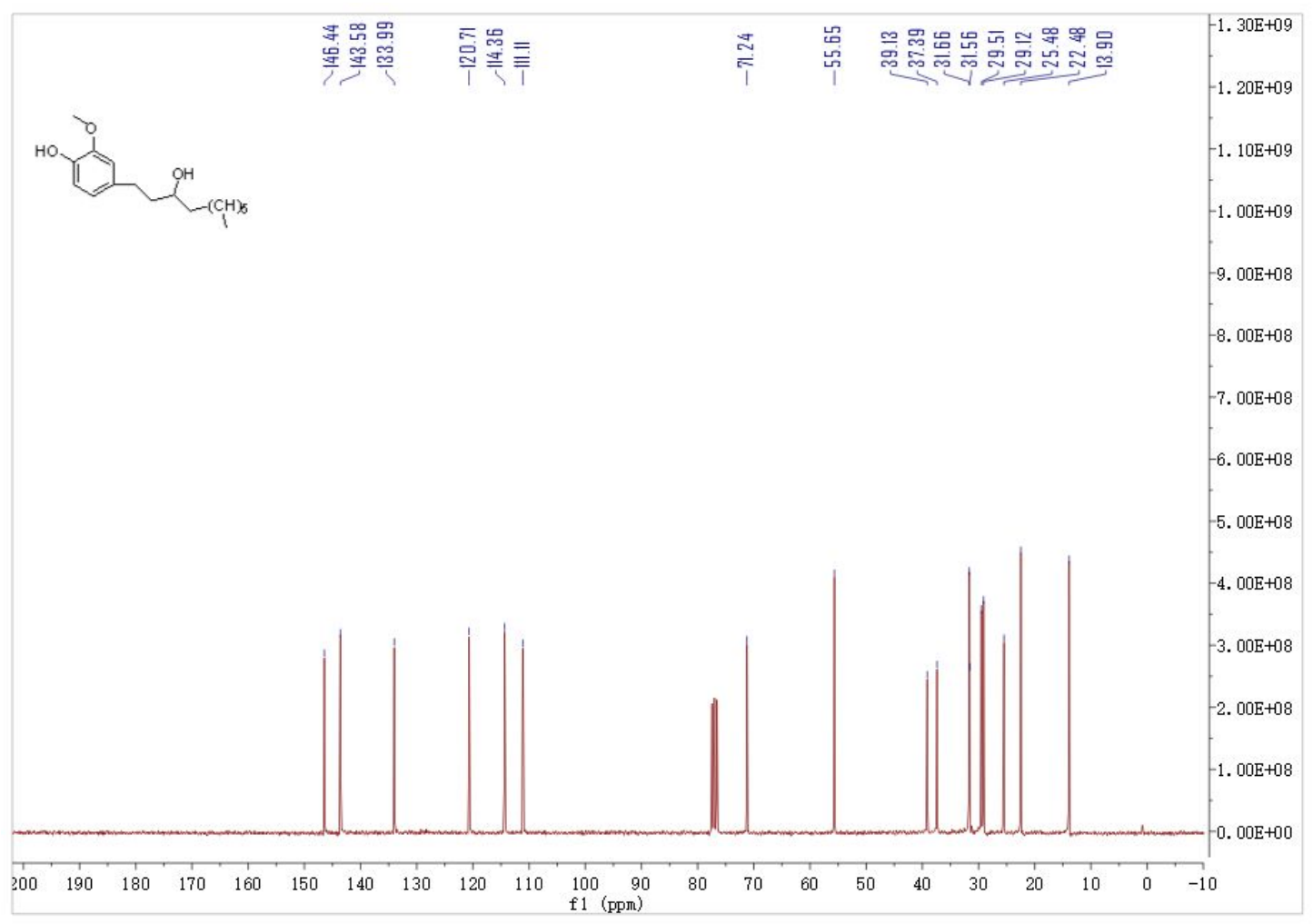


2) The purity value of Compound 1

\begin{tabular}{cc}
\hline Column & YMC-C18 \\
\hline Column size & $4.6 \times 250 \mathrm{~mm}$ \\
Injection & $5 \mu \mathrm{L}$ \\
Mobile phase & Acetonitrile $/ \%$ aqueous solution of \\
& $\mathrm{TFA}=60 / 40(\mathrm{v} / \mathrm{v})$ \\
Flow rate & $1.0 \mathrm{~mL} / \mathrm{min}$ \\
Wave length & $210 \mathrm{~nm}$ \\
Temperature & $25{ }^{\circ} \mathrm{C}$ \\
Running time & $30 \mathrm{~min}$ \\
\hline
\end{tabular}

Sample structure:<smiles>CCCC(=O)CCc1ccc(O)c(OC)c1</smiles>

$<$ Column Performance Report $>$

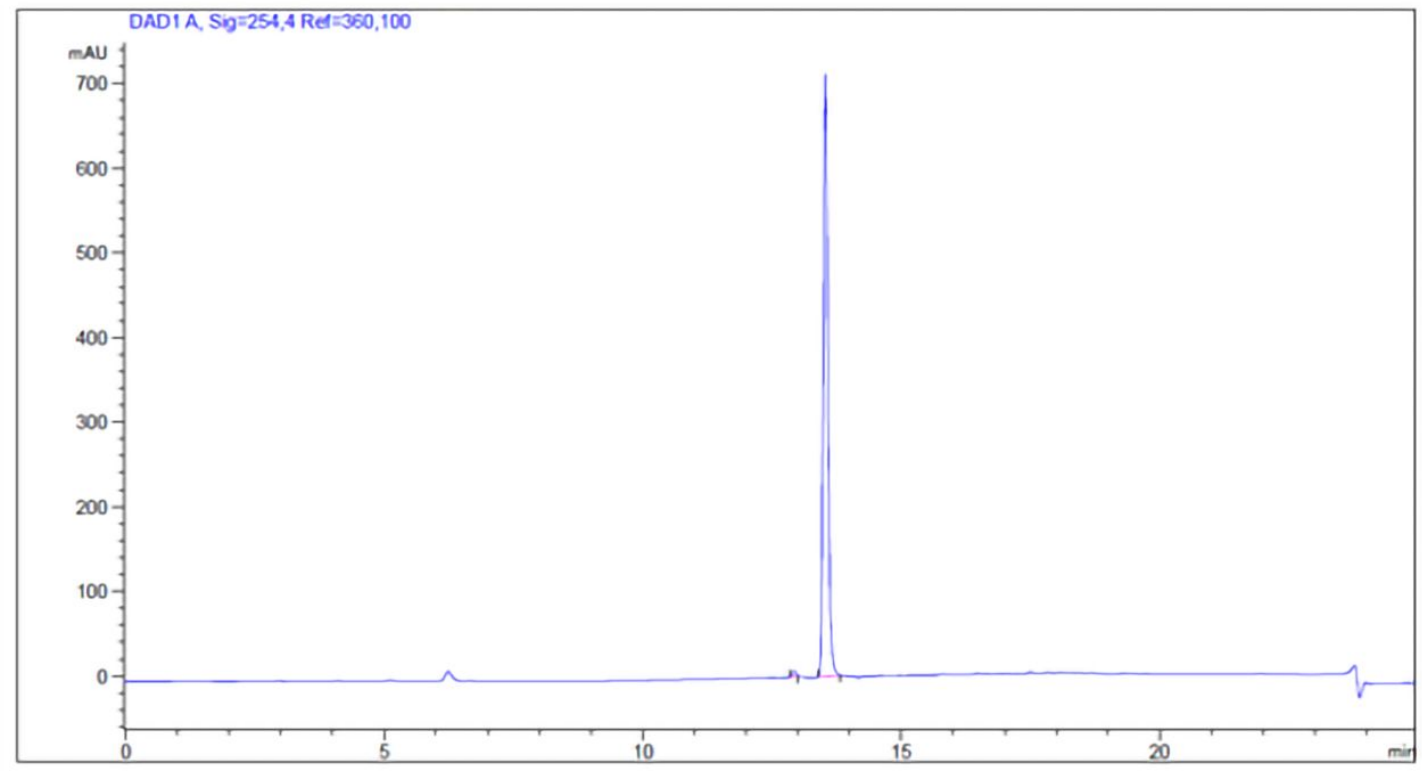

Signal1: DAD1 A, Sig=254,4 Ref $=360,100$

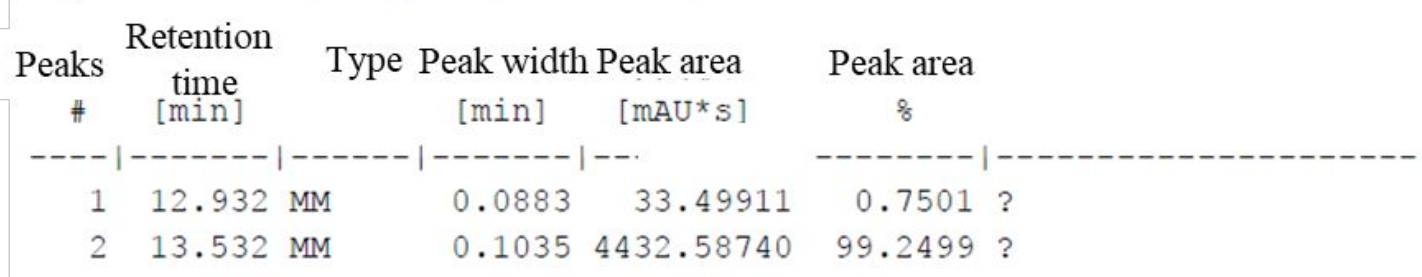

Total :

4466.08651 